I'he Keciprocators of 'I'wo Uonics discussed Geometrically. By. J. W. Russeli, M.A. Received May 8th, 1895. Communicated May 9th, 1895.

\title{
General Construction.
}

1. If two conics $a$ and $\beta$ have a common pole and polar, we can construct a conic $\Gamma$ with respect to which they are reciprocal in the following way :-

Let $U$ bo the common pole; and let the common polar $u$ cut $\alpha$ in $A, A^{\prime}$, and $\beta$ in $B, B^{\prime}$. T'ake $X, X^{\prime}$ the double points of the involution $\left(A B, A^{\prime} B^{\prime}\right)$. Let any tangent $q$ of a cut $A A^{\prime}$ in $N$. Let the line joining $U$ to the fourth harmonic $N^{\prime}$ of $N$ for $X X^{\prime}$ cut $\beta$ in $Q$. Let $Q X$ cut $q$ in $M$. Take $l R$ so that $(Q M, X R)$. is harmonic. Now take. $\Gamma$ as the conic touching $U X$ at $X, U X^{\prime}$ at $X^{\prime}$, and passing through $R$.

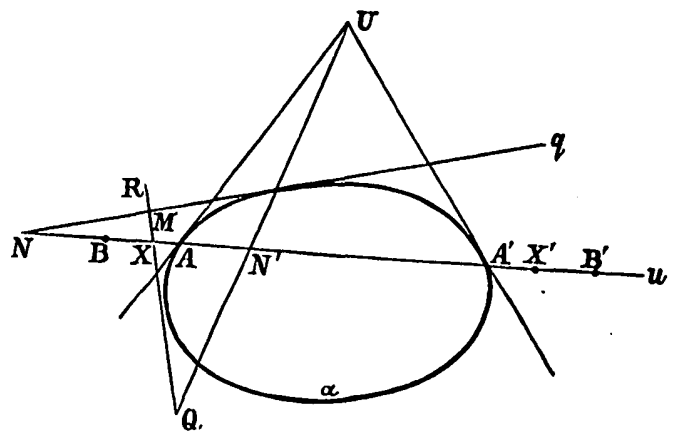

Fit. 1.

Then $A A^{\prime}$ is the reciprocal of $U$. Hence the reciprocal of $B$ passes. through $U$; and also through $A$, since $\left(B A, X X^{\prime}\right)$ is harmonic. Hence $U A$ is the reciprocal of $B$; so $U B$ of $A, U A^{\prime}$ of $B^{\prime}$ and $U B^{\prime}$ of $A^{\prime}$. Hence the reciprocal of $a$ is a conic which touches $U B$ at $B$, and $U B^{\prime}$ at $B^{\prime}$. Now, since $\left(N N^{\prime}, X X^{\prime}\right)$ is harmonic, the reciprocal of $N$ is $U Q$; hence the reciprocal of $Q$ passes through $N$; and also through. $M$ since $(Q M, X R)$ is harmonic. Hence $q$ is the reciprocal of $Q$. Hence the reciprocal of $\alpha$ also passes through $Q$, and is therefore $\beta$.

2. It is convenient to call $\Gamma$ a reciprocator of $a$ and $\beta$.

Since we might have taken $X, X^{\prime}$ as the double points of the invo- 
lution $\left(A B^{\prime}, A^{\prime} B\right)$, and also have taken $Q$ as the other point in which : $U N^{\prime}$ meets $\beta$, the above construction gives four solutions of the problem if $A, B, A^{\prime}, B^{\prime}$ are distinct.

Notice that in all cases the reciprocators $\Gamma$ constructed as above will have a self-conjugate triangle in common with $a$ and $\beta$. For let $V, W$ be the double points of the involution $\left(A A^{\prime}, B B^{\prime}\right)$. Now $X, X^{\prime}$ are the double points of the involution $\left(A B, A^{\prime} B^{\prime}\right)$. Hence $\left(A A^{\prime}\right.$, $\left.B B^{\prime}, X X^{\prime}\right)^{\prime}$ is an involution. Hence $V, W$, being the double points of this involution, are harmonic with $X, X^{\prime}$. Hence $V, W$ are conjugate for $\alpha, \beta$, and $\Gamma$.

\section{Particular Oases.}

3. If the two conics intersect in four distinct points, we may take $U$ at any vertex of the common self-conjugate triangle. Then $A, A^{\prime}$,. $B, B^{\prime}$ will be distinct, and we get four solutions.

If the two conics touch $a$ at $A$ and have two distinct intersections. $D, E$, there is one position of $U$, viz., the intersection of $a$ with $D E$; and the common polar $u$ of $U$ is the line joining $A$ to the intersection

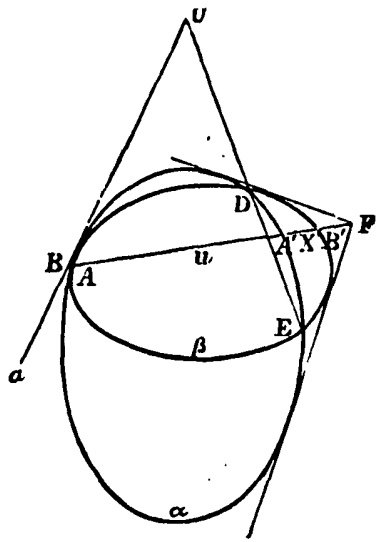

Fig. 2.

of the distinct common tangents. Here $A, B$ coincide. If we take the involution $\left(A B, A^{\prime} B\right), X$ coincides with $A$ and $B$, and $X^{\prime}$ is such that $\left(A X^{\prime}, A^{\prime} B^{\prime}\right)$ is harmonic. We cannot take the involution ( $A B^{\prime}$, $\left.A^{\prime} B\right)$; for no two points can be found which are harmonic both with $A, A^{\prime}$ and with $A, B$. Hence there will be two solutions.

If the two conics have double contact, we may take $U$ at the common pole., Then $A, B$ coincide, and so do $A^{\prime}, B^{\prime}$. If we take the. 
involution $\left(A B, A^{\prime} B^{\prime}\right), X$ is at $A$ and $X^{\prime}$ at $A^{\prime}$; and $\Gamma$ has double contact with $a$ and $\beta$. This gives two solutions. If we take the involu-

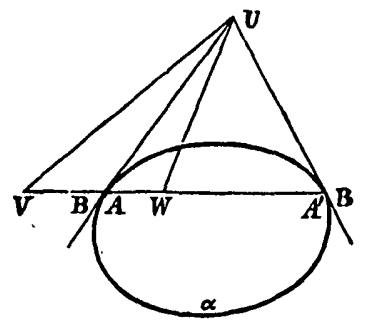

Fia. 3.

tion $\left(A B^{\prime}, A^{\prime} B\right), X$ and $X^{\prime}$ are any two points which divide $A A^{\prime}$ harmonically ; and we get a doubly infinite set of solutions.

If the two conics have four-point contact, we may take $U$ at any point on the common tangent; for any such point has the same polar

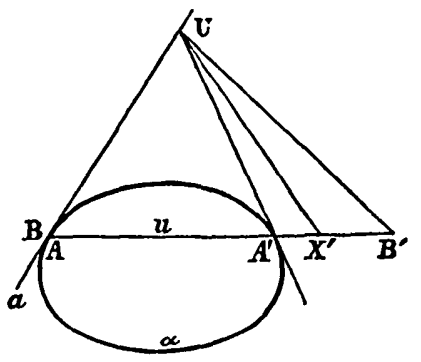

FIG. 4.

for both conics. Hence, as in the previous case, there is apparently a doubly infinite set of solutions (but see $\$ 12$ ).

\section{Exceptional Oonstruction.}

4. If the conics have three-point contact and a distinct intersection, the aljove method fails; for then the conics have no common pole and polar. Let $C$ be the point of contact, and $K$ the distinct intersection. Let the common tangent $k$ meet the tangent $c$ at $C$ in $D$, meet $O K$ in $L$, and touch the conics $a$ and $\beta$ in $A$ and $B$. Let the tangent $b$ at $K$ to a cut $k$ in $M$. 'Take $X$, the fourth harmonic of $C$ for $K, L$. Let $M \Gamma X$ cut $K B$ in $N$, and take $P$ such that $(M \Gamma N, X P)$ is harmonic. 
Now take as $\Gamma$ the conic which touches $c$ at $C$, touches $D X$ at $X$, and passes through $P$.

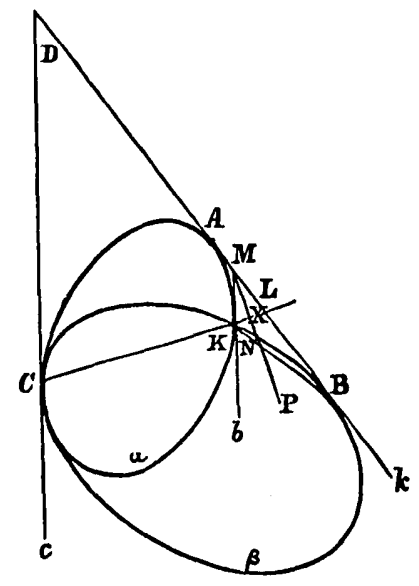

FIG. 5.

Then the reciprocal of $D$ is $C X$. Hence the reciprocal of $K$ is $k$ for $(C X, K L)$ is harmonic. But $K$ is on $a$ and $k$ touches $a$; hence $k$ touches and $K$ is on $\boldsymbol{\alpha}^{\prime}$, the reciprocal of $\alpha$ for $\Gamma$. Now $M$ is on $k$. Hence its reciprocal passes through $K$; and also through $N$, since $(M N, X P)$ is harmonic. Hence $K N$ is the reciprocal of $M$. Hence $B$ is the reciprocal of $M K$ which touches $a$. Hence $B$ is on $\alpha^{\prime}$. Also since $a$ and $\Gamma$ touch at $C, \alpha^{\prime}$ touches both at $O$. Hence $\alpha^{\prime}$ and $\beta$ coincide; for each touches $c$ at $C$, touches $k$ at $B$, and passes through $K$.

Notice that in this case. $\Gamma$ has three-point contact with $\alpha$ and $\beta$ at $\sigma$. For, if $\alpha$ and $\Gamma$ hnve not three-point contact, they have a common pole and polar; hence $\alpha$ and $\beta$ have a common pole and polar; which is not true.

\section{The above Solution is Exhaustive.}

5. We have now shown that in every case there is at least one solution of the problem. Let us next consider whether there are any solutions other than those already found.

Take first the exceptional case in which the conics have threepoint contact. Here the conics $\alpha$ and $\beta$ touch at $O$ only. Hence $\Gamma$ must touch both $\alpha$ and $\beta$ at $O$, for coincident common points must reciprocate into coincident common tangents. Again, the unique intersection $K$ must reciprocate into the unique common tangent $k$ Hence $X$ is known, and then $P$. Hence there is no other solution. 
6. Take the case in which the conics $\alpha$ and $\beta$ intersect in four distinct points. Then $a$ and $\Gamma$ must also intersect in four distinct points, for, if $\alpha$ and $\Gamma$ touch, $\alpha$ and $\beta$ touch. Hence $\alpha$ and $\Gamma$ have one und only one common self-conjugate triangle $U V W$; and this reciprocates into itself and into a triangle which is self-conjugate for $\beta$ and $\Gamma$. Hence $U V W$ must be the common self-conjugate triangle of $a$ and $\beta$. Hence $a, \beta, \Gamma$ have the same common self-conjugate triangle. Hence the polar of $U$ for $\Gamma$ must be $V W$, i.e., we must tako the polar of $U$ for $a$ and $\beta$ as the reciprocal of $U$. Hence the positions of $X, X^{\prime}$ are known. Also the reciprocal of $q$ which passes through $N$ and touches a must lie on the reciprocal of $N$ (viz., $U N^{\prime}$ ), and also on $\beta$. Hence $Q$ is known, and then $R$. Hence there are four und only four reciprocators.

Take the case in which $a$ and $\beta$ touch at $A$, and intersect in two distinct points $D, E$. Then the line $D E$ must reciprocate into $F$, the intersection of distinct common tangents. Also $A$ must reciprocate into $a$. Hence $U$ must reciprocate into $A F$.

Take the case in which $\alpha$ and $\beta$ touch at $A$ and $A^{\prime}$. Then $A$ must reciprocate into $U A$ or $U A^{\prime}$, and $A^{\prime}$ into $U A^{\prime}$ or $U A$. In either case $U$ reciprocates into $A A^{\prime}$.

T'ake, lastly, the case in which the conics have four-point contact at $A$. Then $A$ must reciprocate into $a$, the tangent at $A$. Hence the reciprocal of any point $U$ on $a$ must reciprocate into some line $u$ through $A$, say $d A^{\prime} B$. Then the reciprocal of $A^{\prime}$ on $a$ and $u$ is the tangent to $\beta$ from $U$, i.e., is $U B^{\prime}$. Hence $u$ is the polar of $U$ for $\beta$, and therefore for $a$ also.

Hence in all cases we have obtained every reciprocator.

\section{Self-reciprocal Oonics.}

7. Let us next inquire for what conics a given conic is its own -reciprocal.

Let the point $A$ on the conic $a$ be the reciprocal of the tangent $a$ of the conic $a$. Then the tangent $a^{\prime}$ at $A$ is the reciprocal of the point of contact $A^{\prime}$ of $a$. Hence $\left(A A^{\prime} B B^{\prime} \ldots\right)$ of poles $=\left(a a^{\prime} b b^{\prime} \ldots\right)$ of polars $=\left(A^{\prime} A B^{\prime} B \ldots\right)$ of points of contact. Hence $\left(A A^{\prime}, B I{ }^{\prime}, \ldots\right)$ is an involution. Hence $A A^{\prime}, B B, \ldots$ meet in a fixed point, $O$ say. Now let $A$ be one, $E$, of the points of contact $E, F$ of tangents from $O$. Then the reciprocal of $E$ is the tangent at $E$. Hence the re:quired reciprocator $\Gamma$ touches $O E$ at $E$. Similarly, $\Gamma$ touches $O F$ at $F$. Hence $a$ and $\Gamma$ have double contact. 
Let $A E$ cut $A^{\prime} F$ in $P$, and let $A F$ cut $A^{\prime} E$ in $P^{\prime}$. Then $P$ and $P^{\prime}$ are on $\Gamma$. For, if $a$ cut $A F^{\prime}$ in $L$ and $E F$ in $G$,

$$
\left(A L, F P^{\prime}\right)=A^{\prime}\left(A L, T P^{\prime}\right)=(I I G, F E),
$$

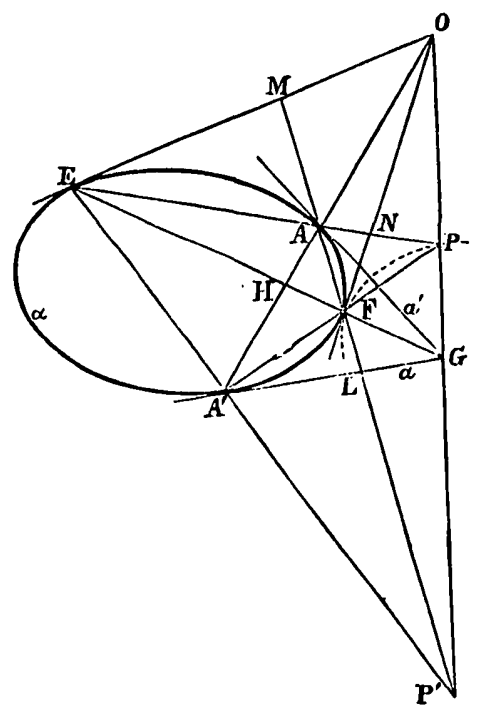

Fig. 6.

which is harmonic, for $G$ is the pole of $A A^{\prime}$. Now $a$ is the reciprocal of $A$, and $F$ is on $\Gamma$. Henco $P$ is on $\Gamma$, and similarly. $P$.

We have still to verify that such a conic exists. Describe a conic $\Gamma^{\prime}$ touching $O E$ at $L, O F^{\prime}$ at $F '$, ind passing through $I^{\prime}$. 'I'lien the reciprocal of $a$ for this conic touches $O E$ at $E$, und $O H^{\prime}$ at $r$. Now, since $\left(\Lambda L, F P^{\prime}\right)$ is harmonic, the reciprocal of $A$ prisses through $L$. Also $A A^{\prime}$ is the reciprocal of $G$, sinco $\left(G I I, F^{\prime} L^{\prime}\right)$ is hamonic. Hence the reciprocal of $A$ passes through $G$. Ilence $a$ is the reciprocal of $A$. Hence the reciprocial of $a$ tonches $O B$ at $K, O F$ at $F '$, and also touches $a$; and hence is $a$. Irence the reciprocial of $a$ for $\Gamma^{\prime}$ is a.

Also the recipecocal of $\Gamma^{\prime}$ for $a$ is $\Gamma^{\prime}$. First notice that $P$ is on tho conic $\Gamma^{\prime}$ by the first part of the above proof. Also that $P P^{\prime}$ passes through $O$ (and also through $G$ ) sinces $P P^{\prime}, \quad L^{\prime}$ and $A A^{\prime}$ are tho diagronials of the sime quithilateral. Now $T^{\prime \prime}$ is generated from a by the intersection of $l^{\prime} A^{\prime}$ and $A F$, and $\alpha$ is generated from $\Gamma^{\prime}$ by tho intersection of $E P^{\prime}$ and $P F^{\prime}$. Honce $\Gamma^{\prime}$ is solf-reciprocal for $\alpha$. 
8. Such conics may be called a pair of self-reciprocal.conics, each being its own reciprocal for the other. The fundamental property of self-reciprocal conics is that they are in harmonic homology, taking $E$ as pole and $O F$ as axis, or $F$ as pole and $O L$ as axis. For

$$
(E N, A P)=O(E N, A P)=(E F, H G) ;
$$

so for $F$. The simplest definition is that they have double contact, and have, at each point of contact, equal and opposite curvatures. For, since ( $M F, A P^{\prime}$ ) is harmonic, $A F: P P^{\prime}:: M M A: M P^{\prime}:: O F: O F$, when $A$ and $P^{\prime}$ coincide with $F$. Hence $A F=F P^{\prime}$ ultimately, and similarly $A^{\prime} F=F P$. And the angles $A F^{\prime} A^{\prime}$ and $P^{\prime} F P$ aro equal. Hence the triangles $A I^{\prime} A^{\prime}$ and $P^{\prime} F^{\prime} P$ are ultimately equal in all respects. Hence the curvatures of $a$ and $\Gamma$ at $F$ are equal and opposite; and similarly at $E$. The above proof does not apply if $F$ is at infinity, but in this case the proposition respecting curvature is nugatory.

\section{Connexion between the Reciprocators.}

9. Let us now study the connexion hetween the various conics $\Gamma$ for which the same two conics $\alpha$ and $\beta$ are reciprocal.

We notice that the reciprocator's always occur in pain's which touch at two points $X$ and $X^{\prime}$, say, and that mother reciprocator cannot touch both of these conics at $X, X^{\prime \prime}$. We shall now prove that the two conics $\Gamma_{1}, \Gamma_{2}$ forming such a pair aro self-reciprocal. For reciplocate for $\Gamma_{1}$. T'lien a reciprocates into $\beta$ and $\beta$ into a. $\Gamma_{1}$ reciprocates into itself. Also $\Gamma_{2}$ reciprocates iuto a reciprocator; for the proposition that $a$ and $\beta$ are reciprocal for $\Gamma_{3}$ reciprocates into the propusition that $\beta$ and $\alpha$ are reciprocal for tho reciprocal $\Gamma_{2}^{\prime}$ of $\Gamma_{2}$. Hence $\Gamma_{2}^{\prime}$ is a reciprocator having double contact with $\Gamma_{1}$ at $X, X^{\prime}$. And the reciprocal for $\Gamma_{1}$ of no conic except $\Gamma_{1}$ can coincide with $\Gamma_{1}$. Henco $\Gamma_{2}^{\prime}$ coincides with $\Gamma_{2}$, i.e., $\Gamma_{1}$ and $\Gamma_{2}$ are self-réciprocal.

10. If the two conics intersect in four distinct points, then each of tho four conics $\Gamma_{1}, \Gamma_{2}, \Gamma_{3}, \Gamma_{1}$ is self-reciprocal for every other. Tor let $X_{1}, X_{1}^{\prime}, X_{2}, X_{3}^{\prime}$ be the doublo points of the above constinction on $V W ; Y_{1}, Y_{1}^{\prime}, Y_{2}, Y_{2}^{\prime}$ those on $W U$; and $Z_{1}, Z_{1}^{\prime}, Z_{3}, Z_{2}^{\prime}$ those on $U V$, where $U V I V$ is the common solf-conjugate triungle of $a$ and $\beta$. Thet $\Gamma_{1}, \Gamma_{3}$, touch $U X_{1}, U X_{1}^{\prime}$ at $X_{1}, X_{1}^{\prime}$, and let $\Gamma_{3}, \Gamma_{4}$ tonch $U X_{3}, U X_{2}^{\prime}$ at $X_{2}, X_{2}^{\prime}$. 'l'hen $\Gamma_{1}, \Gamma_{2}$ atce self-reciprocal, and so are $\Gamma_{3}, \Gamma_{s}$. Now two of tho four touch $V Y_{1}, V Y_{1}^{\prime}$ at $Y_{1}, Y_{1}^{\prime}$; but $\Gamma_{1}, \Gamma_{3}$ cannot touch again, nor can $r_{y}, r_{4}$. Hence $\Gamma_{1}$ must tonch $\Gamma_{3}$, or $\Gamma_{2}$ touch $r_{3}$. 'J'hen $\Gamma_{2}$ touches $\Gamma_{4}$, or $\Gamma_{1}$ touches $\Gamma_{3}$ at $Y_{2}, Y_{2}^{\prime \prime}$. Ifence $\Gamma_{1}, I_{y}^{\prime}$ are self-reciprocal, 
and so are $\Gamma_{2}, \Gamma_{4}$. Similarly, $\Gamma_{1}, \Gamma_{4}$ touch on $U V$, and so do $\Gamma_{2}, \Gamma_{8}$. Hence $\Gamma_{1}, \Gamma_{4}$ are self-reciprocal, and so are $\Gamma_{2}, J_{s}$. Hence every one of the conics $\Gamma_{1}, \Gamma_{2}, \Gamma_{8}, \Gamma_{4}$ is self-reciprocal for every other.

If the two conics touch and intersect in two points, the two conics $\Gamma_{1}, \Gamma_{2}$ are self-reciprocal; for they havo double contact.

11. If the two conics $\alpha$ and $\beta$ have double contact at $A$ and $A^{\prime}$, the two reciprocators $\Gamma_{1}$ and $\Gamma_{2}$ which have double contact with them at $A$ and $A^{\prime}$ ar'e, as before, self-reciprocal. Let $\Gamma_{8}$ and $\Gamma_{8}$ be the reciprocators touching at $X, X^{\prime}$, where $X, X^{\prime}$ are harmonic with $A, A^{\prime}$; these also are, as before, self-reciprocal. Take $V, W$ the double points of the involution $\left(A A^{\prime}, X X^{\prime}\right)$. Then $V, W$ are conjugate for $a, \beta, \Gamma_{1}, \Gamma_{2}$, $\Gamma_{8}, \Gamma_{8}$. Hence $U V W$ is a self-conjugate triangle for these conics. Also no other reciprocator has $U V W$ as a self-conjugate triangle. For suppose the reciprocator's $\gamma_{3}^{\prime}, \gamma_{4}^{\prime}$ touching at $x, x^{\prime}$ have $U V W$ as a self-conjugate triangle. 'T'hen, $V, W$ are larmonic with $x, x^{\prime}$, which are harmonic with $A, A^{\prime}$. Hence $x, x^{\prime}$ coincide with $X, X^{\prime}$; for these are also harmonic with both $A, A^{\prime}$ and $V, W$. Now the four reciprocators obtained by using $V$ or $W$ as pole have also $U V W$ as a selfconjugate triangle (see $\S 2$, end). Hence these conics are the same as $\Gamma_{1}, \Gamma_{2}, \Gamma_{8}, \Gamma_{4}$. Hence, as in $\S 10$, they are self-reciprocal in pairs. Hence we conclude that each of the variable conics $\Gamma_{s}, \Gamma_{4}$ has double contact with the fixed conice $\Gamma_{3}, \Gamma_{2}$ both on $U V$ and on $U W$.

12. Finally, if the conics $a$ and $\beta$ have four-point contact, the reciprocators consist of one conic $\Gamma^{\prime}$ and a system of conics selfreciprocal for $\Gamma^{\prime}$. For (see Fig. 4), the conic $\Gamma^{\prime}$ baving four-point contact with both $\alpha$ and $\beta$ at $A$, and passing through $X^{\prime}$ is a reciprocator. For the tangent at $X^{\prime}$ is $U X^{\prime}$, since the tangents at $A^{\prime}$ and $X^{\prime}$ to $a$ and $\Gamma^{\prime}$ meet on $A U$; hence $A A^{\prime}$ is the reciprocal of $U$. Honce the reciprocal of $U A^{\prime}$ is $B^{\prime}$. Hence the reciprocal of a for $\Gamma^{\prime}$ is a conic having four-point contact with $\beta$ at $A$, and passing through ' $B^{\prime}$, i.e., is $\beta$. Also $\Gamma^{\prime}$ is one of the reciprocators belonging to $A A^{\prime}$, for it is a reciprocator and touches $U X^{\prime}$ at $X^{\prime}$ and $U A$ at $A$. And the other is self-reciprocal for $\Gamma^{\prime}$.

13. We can extend the above construction to two quadrics. For instance, take the case in which the two quadrics have a common pole and polar, $U$ and $u$. Let the quadrics bo $\phi$ and $\psi$, and lot their sections by the plane $u$ be $\alpha$ and $\beta$. Take any reciprocator $\gamma$ of the conics $\alpha$ and $\beta$. Let any tangent plane $q$ of $\phi$ cut $u$ in the line $u$. YoL. XXVI. - NO. 529. 
Let the line joining $U$ to the pole $N^{\prime}$ of $n$ for $\gamma$ cut $\psi$ in the point $Q$. Let the line joining $Q$ to any point $X$ on $\gamma$ meet $q$ in $M$, and take the point $R$ so that $(Q M I, X R)$ is harmonic. Then a reciprocator of the quadrics $\phi$ and $\psi$ is the quadric $\Gamma$ which passes through $R$ and touches the cone joining $U$ to $\gamma$ along $\gamma$.

For let $\phi^{\prime}$ bo the reciprocal of $\phi$ for $\Gamma$. Then, since $\phi$ touches the cone joining $U$ to $\alpha$ along $\alpha, \phi^{\prime}$ touches the cone joining $U$ to $\beta$ along $\beta$; for $U$ is tho reciprocal of $u$. Now $\left(Q\right.$ lies on $U N^{\prime}$. Also $N^{\prime}$ is the reciprocal of $U n$. Hence the reciprocal of $(Q$ passes through the intersection of $u$ and $U u$, i.e., throngh $n$; and it passes through $M$, since $(() M, X I R)$ is harmonic; hence it is $q$. But $q$ touches $\phi$; hence $Q$ is on $\phi^{\prime}$. Hence $q^{\prime}$ is $\psi$.

Notice that, since ( $)$ may have two positions, we gret twice as many reciprocators of the quadrics as there are of the conics $a$ and $\beta$.

By A. E. Jolurfe. Received May 8th, 1895.

Communicated Maly 9 th, 1895.

The following analytical investigation was undertaken with the object of contirming MIr. Russell's statements as to the number of the conics $\Gamma$ in the diffenent cases, concerning which some doubts had been expressed. For this reison the result in $\$ 4$ is obtained by rigorous analysis insteat of being assmmed at geometrically obvious. 'llie result, of $\$ 2$ and the existence of the intinite systems in the cases of donlle and four-point contact were first obtained as below by means of analysis. It is hoped that the aulditional matter contained in $\S 7$ ancl onwards may be not withont interest.

1. In the case of four distinct intersections, the equations of the two conics may le written

$$
\begin{aligned}
S & \equiv x^{3}+y^{2}+z^{3}=0, \\
S^{\prime} & \equiv p \cdot x^{2}+q y^{2}+z^{2}=0 .
\end{aligned}
$$

Take the equation of $r$ in the most genceal form possible, viz.,

$$
a z^{2}+b y^{2}+c z^{3}+2 j y z+2 y z z+2 l u x y=0 .
$$

